\title{
Downregulation of telomerase maintenance-related $A C D$ expression in patients undergoing immunosuppresive therapy following kidney transplantation
}

\author{
AGNIESZKA WITKOWSKA ${ }^{1}$, BARBARA STRZALKA-MROZIK ${ }^{2}$, ALEKSANDER OWCZAREK ${ }^{3}$, JOANNA GOLA ${ }^{2}$, \\ URSZULA MAZUREK $^{2}$, WLADYSLAW GRZESZCZAK ${ }^{1}$ and JANUSZ GUMPRECHT ${ }^{1}$ \\ ${ }^{1}$ Department of Internal Medicine, Diabetology and Nephrology, Medical University of Silesia, 41-800 Zabrze, Silesia; \\ ${ }^{2}$ Department of Molecular Biology; ${ }^{3}$ Division of Statistics, Medical University of Silesia, 41-200 Sosnowiec, Silesia, Poland
}

Received August 30, 2014; Accepted July 23, 2015

DOI: $10.3892 /$ etm.2015.2785

\begin{abstract}
Chronic administration of immunosuppressants has been associated with long-term consequences, including a higher risk of neoplasm development. The processes regulating telomere function exert a major influence on human cancer biology. The present study aimed to assess the effect of immunosuppressive therapy on the expression of genes associated with telomere maintenance and protection in patients following renal transplantation. A total of 51 patients that had undergone kidney transplantation and 54 healthy controls were enrolled in the study. The 51 transplant patients received a three-drug immunosuppressive regimen consisting of cyclosporine A, prednisone and mycophenolate mofetil. In stage 1 of the study, the expression profiles of 123 transcripts, which represented 70 genes, were assessed in peripheral mononuclear blood cells using an oligonucleotide microarray technique in 8 transplant recipients and 4 healthy control subjects. Among the analyzed transcripts, the expression levels of 4 differed significantly between the studied groups; however, only the $A C D$ (adrenocortical dysplasia homolog) gene, encoding the telomere-binding protein POT1-interacting protein 1 (TPP1), was sufficiently specific for telomere homeostasis. The expression of $A C D$ was downregulated in transplant recipients (fold change, 2.11; $\mathrm{P}=0.006$ ). In stage 2 of the study, reverse transcription-quantitative polymerase chain reaction analysis of $A C D, D K C l$ and $h T E R T$ mRNA was conducted for all transplant patients and control subjects. The results confirmed the downregulation of the $A C D$ gene in patients that had received immunosuppressive therapy $(\mathrm{P}=0.002)$. The results of the present study indicate that the downregulation of $A C D$
\end{abstract}

Correspondence to: Dr Agnieszka Witkowska, Department of Internal Medicine, Diabetology and Nephrology, Medical University of Silesia, 3 Maja 13/15, 41-800 Zabrze, Silesia, Poland

E-mail: witkowskaaga@op.pl

Key words: telomerase, TPP1, ACD gene, immunosupression, kidney transplantation gene transcription, and thus TPP1 protein expression, may enhance the capacity for cell immortalization, despite normal levels of other key telomere maintenance factors, in patients undergoing immunosuppressive therapy. Furthermore, the results indicate that TPP1 has potential for use as an early clinical marker and/or therapeutic target for cancer in patients following organ transplantation.

\section{Introduction}

Solid organ transplant recipients are at an elevated risk of cancer development. Compared with the general population, cancers develop more rapidly, occur earlier and metastasize more widely in this group of patients (1). Following kidney transplantation the risk of cancer development increases 2-4-fold; thus, following cardiovascular disease, kidney transplantation is a major cause of morbidity (2). Certain types of cancer are particularly over-represented among the transplant patient population; for example, marked increases in incidence have been observed in oncovirus-related tumors, such as Kaposi's sarcoma, skin cancer and lymphoma, which are associated with a $>20$-fold increase in risk. In addition, the rate of kidney malignancies is increased 15-fold in transplant patients compared with the general population (3). Common malignancies, such as lung, ovarian, colon or gastric cancer, have an incidence that is $\sim 2$-fold higher, while the incidence of leukemia, liver, gynecological, bladder and testicular tumors increases $\sim 5$-fold following renal transplantation (3). The increased risk of carcinogenesis observed in patients following renal transplantation is the result of conventional risk factors (genetic, immune or environmental), in addition to risk factors specific to transplant recipients (primarily immunosuppressive therapy and, in certain cases, oncogenic viruses) (1); however, immunosuppressive therapy appears to be the major factor responsible for the increased cancer incidence following transplantation (4).

The development of cancer is a multistage process involving numerous mutations and/or chromosomal aberrations; therefore, cancer is regarded as a disease of genomic instability (5). The majority of the genetic aberrations that are characteristic of cancer can be initiated by telomere dysfunc- 
tion $(6,7)$. Telomeres are nucleoprotein structures that protect the ends of eukaryotic chromosomes. The formation of a telosome, which is a DNA loop structure with a number of associated proteins, including the six core factors telomeric repeat-binding factor 1 (TRF1), TRF2, telomeric repeatbinding factor 2 -interacting protein 1, TERF1-interacting nuclear factor 2 , protection of telomeres protein 1 (POT1) and POT1-interacting protein 1 (TPP1), protects the very end of a telomere against a DNA break, preventing chromosomal end-to-end fusions, misrepair and degradation (6,8-11). Telomere shortening promotes genome instability, and shortened telomeres have been reported to be common and prevalent early genetic alterations in cancer initiation $(12,13)$. Furthermore, the stability of telomere length determines immortalization, which is the obligatory step of cancer development (14). In immortal cells, a balance is maintained between the loss of telomeric DNA due to degradation or incomplete replication and telomere elongation, which is performed by a DNA polymerase known as telomerase (15). Human telomerase is composed of telomerase RNA (hTR) and a catalytic subunit, telomerase reverse transcriptase (hTERT) $(16,17)$. Telomerase is believed to be strongly repressed in normal human somatic tissues, but reactivated in $85-90 \%$ of human cancer tissues $(18,19)$; however, a previous study has indicated that, even in the presence of proficient telomerase activity and normal telomere length, telomere-associated proteins have an important role in cancer (9).

As telomere dysfunction has been implicated in leukemia and cancer development $(9-11,20)$, the various factors involved in telomere maintenance may emerge as potential cancer markers and/or therapeutic targets. The identification of novel cancer markers is particularly important for patients receiving immunosuppressive therapy, as these patients are at an increased risk of cancer and require more frequent diagnostic screening for the early identification of cancer. The aim of the present study, therefore, was to assess the effect of immunosuppressive therapy on the expression of genes associated with telomere maintenance and protection in patients following renal transplantation.

\section{Materials and methods}

Study design. In order to establish the factors involved in telomere maintenance and protection that best differentiated between the cases and controls, the study was divided into two stages, as described below. The study was approved by the Bioethics Committee of the Medical University of Silesia (Katowice, Poland) in accordance with the Declaration of Helsinki regarding medical research involving human subjects. The study and its purpose were explained to each participant or their legal guardian, who gave their informed written consent.

In stage 1 of the study, the expression profiles of 123 transcripts, which represented 70 genes involved in telomere maintenance, were selected as described by Witkowska et al (21) and assessed in peripheral mononuclear blood cells (PBMCs) from representatives of the study and control groups using an oligonucleotide microarray technique (HG-U133A array; Affymetrix, Inc., Santa Clara, CA, USA).
In stage 2 of the study, reverse transcription-quantitative polymerase chain reaction (RT-qPCR) analysis of the selected telomere maintenance factors was performed in all patients in the study and control groups.

Subjects. A total of 51 kidney transplantation patients and 54 healthy control subjects were enrolled in the study. Kidney transplant recipients (35 men and 16 women; age, $48.7 \pm 6.8$ years), who had undergone renal transplantation surgery an average of 9.6 years previously, were treated with a three-drug homogenic immunosuppressive regimen, consisting of cyclosporine A (CsA), prednisone and mycophenolate mofetil (MMF) or mycophenolate sodium. Doses were determined based on the patients weight, and all patients underwent serum drug concentration monitoring. Patients with an acute disease were excluded (C-reactive protein-negative). Fifty-four medication-free healthy subjects (11 men and 43 women) were selected for the control group. A total of 7 euglycemic transplant recipients (5 men and 2 women; age, $46.5 \pm 8.6$ years) and 4 healthy controls ( 2 men and 2 women; age, $59.0 \pm 4.5$ years) were selected for microarray assay analysis in stage 1 of the study.

Tissue samples. Venous blood samples were collected and stored in tubes containing EDTA, and a 7.5-ml sample from each patient was centrifuged using Ficoll-Conray density gradient centrifugation (specific gravity, 1.077) for $30 \mathrm{~min}$ at 350 x g (Immuno-Biological Laboratories Co., Ltd., Gunma, Japan), immediately after blood collection. A 10-ml sample was collected from patients selected for microarray assay.

RNA extraction. Total RNA was extracted from the PBMCs using TRIzol ${ }^{\circledR}$ reagent (Invitrogen Life Technologies, Carlsbad, CA, USA) and then treated with DNase I (MBI Fermentas; Thermo Fisher Scientific, Vilnius, Lithuania) according to the manufacturer's instructions. The quality of the extracted RNA was tested electrophoretically using ethidium bromide-stained $0.8 \%$ agarose gel. The results were analyzed and recorded using an electrophoresis documentation system (Fisher Biotec, Perth, WA, Australia) and LabImage-1D software, version 2.7.2 (Kapelan Bio-Imaging GmbH, Leipzig, Germany). The total RNA concentration was measured spectrophotometrically using the Gene Quant II RNA/DNA Calculator (Pharmacia; GE Healthcare, Cambridge, UK).

Oligonucleotide microarray analysis. The analysis of the expression profiles of 123 transcripts, which represented 70 genes (21), was performed using commercially available oligonucleotide microarrays (HG-U133A; Affymetrix, Inc.) according to the manufacturer's instructions and the protocol previously described by Rostkowska-Nadolska et al (22).

$R T-q P C R$ assay. Detection of the expression of $h T E R T, A C D$, $D K C 1$ and $G A P D H$ mRNA was performed using RT-qPCR with a QuantiTect ${ }^{\circledR}$ SYBR ${ }^{\circledR}$ Green RT-PCR kit (Qiagen, Inc., Valencia, CA, USA), according to the manufacturer's instructions, and an Opticon DNA Engine Continuous Fluorescence detector (MJ Research, Inc., Waltham, MA, USA), as described previously (23). All samples were tested in triplicate. GAPDH levels were measured for each sample to exclude 
Table I. Characteristics of primers used for amplification.

\begin{tabular}{|c|c|c|c|}
\hline Gene & Primer sequence & Amplicon length (bp) & $\operatorname{Tm}\left({ }^{\circ} \mathrm{C}\right)$ \\
\hline$G A P D H$ & $\begin{array}{l}\text { Forward: 5'-GAAGGTGAAGGTCGGAGTC-3' } \\
\text { Reverse: 5'-GAAGATGGTGATGGGATTC-3' }\end{array}$ & 226 & 80.0 \\
\hline hTERT & $\begin{array}{l}\text { Forward: 5'-CGCCGCCTGGCTGTACTTTGTC-3' } \\
\text { Reverse: 5'-TTGTTCTCCATGTCGCCGTAGCA-3' }\end{array}$ & 451 & 76.6 \\
\hline$A C D$ & $\begin{array}{l}\text { Forward: 5'-AAAGGTTGGATTGGACTTTCC-3' } \\
\text { Reverse: 5'-GAACGTGAGGCTACGCTGA-3' }\end{array}$ & 60 & 86.2 \\
\hline$D K C 1$ & $\begin{array}{l}\text { Forward: 5'-TCTTCTTTTCCTTCTTGATCAACTG-3' } \\
\text { Reverse: 5'-GCCGAAGCAGCAAAAACT-3' }\end{array}$ & 100 & 82.8 \\
\hline
\end{tabular}

bp, base pairs; Tm, melting temperature.

possible RT-qPCR inhibitors. Oligonucleotide primers that were specific for GAPDH (3) and hTERT (6) were selected on the basis of the published data. Oligonucleotide primers that were specific for $A C D$ and $D K C 1$ were designed using Primer Express $^{\mathrm{TM}}$ software, version 2.0 (Applied Biosystems; Life Technologies, Foster City, CA, USA). Primers were synthesized at the Institute of Biochemistry and Biophysics Polish Academy of Sciences (Warsaw, Poland). The characteristics of the primers used for amplification are presented in Table I. The thermal profile for one-step RT-qPCR was as follows: $\mathrm{RT}$ at $50^{\circ} \mathrm{C}$ for $30 \mathrm{~min}$; denaturation at $95^{\circ} \mathrm{C}$ for $15 \mathrm{~min}$; and 40 cycles of $94^{\circ} \mathrm{C}$ for $15 \mathrm{sec}, 60^{\circ} \mathrm{C}$ for $30 \mathrm{sec}$ and $72^{\circ} \mathrm{C}$ for $30 \mathrm{sec}$. A cycle threshold $(\mathrm{Ct})$, which is the point at which a PCR product is detected above a fixed threshold for the first time, was determined for each sample.

A standard curve method was used to quantify the RT-qPCR results for $h T E R T, A C D, D K C l$ and $G A P D H(24,25)$. Commercially available $\beta$-actin cDNA standards (TaqMan ${ }^{\circledR}$ DNA Template Reagent kit; Applied Biosystems) were used at $0.6,1.2,3.0,6.0$ and $12.0 \mathrm{ng} / \mu$ l to simultaneously detect the expression profile of each of the target genes. For the standards, copy number values were calculated based on $1 \mathrm{ng}$ DNA equaling 333 genome equivalents (Applied Biosystems). Amplification plots for each dilution of a commercially available standard template were used to determine the $\mathrm{Ct}$ values. $\mathrm{Ct}$ values were plotted against the log of the known quantity of $\beta$-actin cDNA copy numbers to generate a standard curve. The standard curves exhibited correlation coefficients of between 0.988 and 0.995 , which indicated a high degree of confidence for the measurement of the copy number of molecules in each sample. Melting curve analysis was used complete each run and confirm the specificity of amplification and the absence of primer dimmers. The RT-qPCR products were separated on $6 \%$ polyacrylamide gels and visualized using silver salts.

Statistical analysis. In stage 1 of the study (for microarray data) statistical analysis was performed using GeneSpring GX software, version 11.0 (Agilent Technologies, Inc., Santa Clara, CA, USA; unpaired t-test with asymptotic P-values corrected using the Benjamini-Hochberg multiple comparison test). RT-qPCR data obtained in stage 2 of the study are presented as the mean \pm standard deviation (t-test). The normality of data distribution was assessed using the Shapiro-Wilk test. Due to abnormal distribution, data were normalized via logarithmic transformation. Additionally, results were adjusted against GAPDH levels using Relative Expression Software Tool (REST) software (26). All calculation was performed using Statistica software, version 9.0 (StatSoft Ltd., Bedford, UK) and REST software (26). $\mathrm{P}<0.05$ was considered to indicate a statistically significant difference.

\section{Results}

Stage 1. Among the transcripts analyzed by microarray, 4 were found to exhibit significantly different expression levels between the transplant patients and control subjects (fold change, $>2.0 ; \mathrm{P}<0.05)$, as shown in Table II. The only gene directly influencing telomere maintenance that significantly differed both study and control groups was ACD. It encodes TPP1, one of the six core proteins in the telosome of the telomeric complex which expression and it was decreased in the study group. The expression of two genes involved indirectly in the regulation of telomerase transcription: TGFBR2 (encodes receptor for TGF beta) that inhibits telomerase by TGFbeta and MYC and MAP3K1gene (encodes kinase MAP1) that influence telomerase transcription by grow factors were increased in study group. Similarly, gene YWHAB that encodes protein taking part in nuclear transport telomerase complex and thus influencing telomerase activity was overexpressed.

Stage 2. Based on the results of the stage 1 microarray analysis and current knowledge regarding the effect of immortalization factors on telomere maintenance $(9,15-17,21)$, three mRNAs were selected for RT-qPCR analysis in stage 2 of the study: i) $h T E R T$, which encodes a catalytic subunit of telomerase and component of the hTERT complex (16); ii) $A C D$, also referred to as telomere binding protein TPP1, which encodes one of the six core proteins of the telosome, mediates the access of telomerase to the telomere and regulates telomerase enzymatic activity; and iii) DKCl (dyskeratosis congenita 1 or dyskerin), a member of the small nucleolar ribonucleoprotein gene family and component of the hTR subunit complex.

The results of the REST software analysis for the hTERT, $A C D$ and $D K C l$ transcripts are shown in Table III. ACD was 
Table II. Results of the stage 1 microarray analysis.

\begin{tabular}{|c|c|c|c|c|}
\hline Gene & P-value & Fold change & Result $^{\mathrm{a}}$ & Association with telomere maintenance regulation \\
\hline$A C D$ & 0.006 & 2.11 & Down & $\begin{array}{l}\text { Encodes one of six core telosome/shelterin proteins } \\
\text { and mediates the access of telomerase to the telomere }\end{array}$ \\
\hline TGFBR2 & 0.020 & 2.42 & Up & Regulation of telomerase transcription \\
\hline$M A P 3 K 1$ & $<0.001$ & 4.02 & Up & Regulation of telomerase transcription \\
\hline$Y W H A B$ & 0.002 & 2.97 & $\mathrm{Up}$ & Nuclear transport of telomerase \\
\hline
\end{tabular}

${ }^{a}$ Expression regulation in transplant recipients versus controls.

Table III. Results of the stage 2 t-test and the REST software analysis.

\begin{tabular}{|c|c|c|c|c|c|c|c|}
\hline \multirow[b]{2}{*}{ Gene } & \multicolumn{2}{|c|}{$\begin{array}{c}\text { t-test } \\
\log (\text { copy number } / \mu 1 \mathrm{RNA})^{\mathrm{a}}\end{array}$} & \multicolumn{5}{|c|}{ REST software analysis } \\
\hline & $\begin{array}{l}\text { Study } \\
\text { group, } \mathrm{n}=51\end{array}$ & $\begin{array}{c}\text { Control } \\
\text { group, } n=54\end{array}$ & $\begin{array}{c}\text { Relative } \\
\text { expression }\end{array}$ & SE & $95 \% \mathrm{CI}$ & P-value & Result $^{\mathrm{b}}$ \\
\hline GAPDH & $4.10 \pm 1.25$ & $4.44 \pm 1.25$ & 1.000 & - & - & - & - \\
\hline$A C D$ & $4.12 \pm 1.09$ & $3.90 \pm 1.18$ & 0.684 & $0.312-1.644$ & $0.088-2.960$ & 0.002 & Down \\
\hline$D K C 1$ & $3.89 \pm 1.27$ & $4.08 \pm 1.15$ & 0.952 & $0.414-1.890$ & $0.138-12.553$ & 0.708 & - \\
\hline hTERT & $2.17 \pm 1.42$ & $2.67 \pm 1.33$ & 1.151 & $0.342-3.935$ & $0.148-12.934$ & 0.389 & - \\
\hline
\end{tabular}

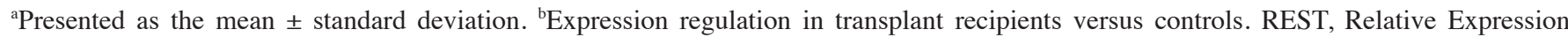
Software Tool; SE, standard error; CI, confidence interval.

downregulated in the transplant recipient group by a mean factor of 0.684 compared with the control group, and the difference between the samples in the two groups was significant $(\mathrm{P}=0.002)$. The differences in the DKCl and hTERT expression levels between the two groups were not significant ( $\mathrm{P}=0.70$ and $\mathrm{P}=0.38$, respectively).

\section{Discussion}

As cellular immortalization plays a key role in chromosome instability and carcinogenesis, knowledge of the mechanisms of telomere dysfunction may be crucial for understanding neoplasm formation and may aid the identification of early clinical markers and/or therapeutic targets for cancer. Furthermore, the influence of major oncological risk factors following renal transplantation and the significantly enhanced incidence of malignancy in transplant patients necessitates the identification of novel cancer markers, particularly for high-risk transplant patients. The aim of the present study, therefore, was to identify a specific differential factor among those involved in cell immortalization and telomere maintenance.

Telomere length homeostasis is a complex process and involves $h T R$ and $h T E R T$ gene amplification, regulation of $h T R$ and $h T E R T$ transcription (mediated directly by transcription factors and indirectly by hormones and growth factors), epigenetic modulation, alternative splicing of $h T E R T$, other post-transcriptional modulations of the primary telomerase subunits and components, regulation of the telomerase complex and hTR complex activities, translocation of those complexes, and interactions between cell cycle regulators and telosome proteins $(8,17,27-29)$. Furthermore, telomere maintenance requires the regulation of various DNA-damage response signals, including the ataxia telangiectasia mutated and ataxia telangiectasia and Rad3-related pathways, homologous recombination and non-homologous end joining (10). In addition, a recent study has suggested that telomerase promotes cell growth via pathways that are not associated with telomere maintenance, and a subset of tumors elongate telomeres through telomerase-independent mechanisms (30).

Due to the complexity of telomere regulation, the aim of the present study was to identify gene candidates specific to telomere biology, as well as the differential factor that had most potential for use as a candidate for testing. The study was, therefore, designed in two stages. In stage 1, differential factors were identified among 70 genes (123 transcripts) involved in telomere maintenance. The genes were selected in accordance with the method described in detail by Witkowska et al (21). Among the analyzed transcripts, 4 were found to exhibit significantly different expression levels between the transplant patients and control subjects; however, only the $A C D$ gene was sufficiently specific for telomere homeostasis. The $A C D$ gene encodes TPP1, one of six core proteins in the telosome or shelterin complex that is involved in the regulation of the access of telomerase to the telomere. In addition to the $A C D$ gene (TPP1 protein), the hTERT and DKCl genes were selected for stage 2 of the study. Although the hTERT and DKCl transcripts were not differential factors in stage 1 , they were selected for RT-qPCR analysis due to their regulatory role in 
the activity of the hTERT and hTR complexes. The results of stage 2 of the study confirmed the downregulation of the $A C D$ gene transcripts, and thus reduced expression of TPP1, in patients undergoing immunosuppressive therapy. By contrast, the expression levels of $h T E R T$ and the hTR complex component $D K C l$ did not differ significantly between the patient and control groups, which may additionally indicate the crucial role played by TPP1 in telomere dysfunction.

TPP1 is one of the six core proteins in the telosome of the telomeric complex that are involved in the maintenance of telomere length and the protection of telomere ends. By interacting with various cell components, TPP1 serves a key function in the assembly and stabilization of this complex and mediates the access of telomerase to the telomere. The role of TPP1 in telomerase recruitment and regulation has been increasingly elucidated in the past 5 years (9). TPP1 in the context of telomere regulation has been studied predominantly in vitro and in vivo in animal models, and there are limited human clinical studies in this field. In addition to the role of TPP1 in recruiting POT1a and POT1b to the chromosome ends and regulating the access of telomerase to the telomere (31), it has been proposed that TPP1 regulates telomerase activity at chromosome ends by direct interaction with telomerase $(32,33)$. It has also been indicated that TPP1 is required for telomere elongation by telomerase (34).

According to a recently proposed model of the mechanisms underlying the initiation and termination of telomerase-mediated telomere elongation in cancer cells, human telomerase binds TPP1 at telomeres during the $S$ phase of the cell cycle and adds $\sim 60$ nucleotides in a single round of extension, after which the telomerase is inhibited by the CST (CTC1, STN1 and TEN1) complex (35). The human CST complex, which has previously been shown to be involved in telomere protection and DNA metabolism, acts to inhibit telomerase activity through primer sequestration and by interacting with the telomerase processivity factor POT1-TPP1. CST compete with POT1-TPP1 for telomeric DNA, and depletion of CST allows excessive telomerase activity, promoting telomere elongation (35).

A previous study by Nakashima et al (36) demonstrated that the combined action of telomerase inhibitor and TPP1 mutations inhibited the growth of HeLa cell lines, and the death of these cells was primarily via an apoptotic mechanism. The results of the study suggested that the inhibition of telomerase-TPP1 binding, possibly combined with moderate inhibition of the telomerase enzyme, could represent an effective anticancer therapy for the $\sim 90 \%$ of human tumors that are telomerase-positive. Furthermore, Yang et al (37) showed in an in vitro study that enhanced TPP1 expression was significantly associated with radioresistance and increased telomere length. It was suggested that increased TPP1 expression in human colorectal cancer cells could have a protective effect on telomeres against DNA damage and confer radioresistance, and that TPP1 could represent a potential target for the radiotherapy of colorectal cancer.

Studies of mouse models with defective TPP1 expression have demonstrated the importance of the telosome in cancer and aging. It has been shown in vivo that TPP1 deletion reduces the binding of TERT to telomeres, as well as telomerase function at chromosome ends. A lack of TPP1 may lead to perinatal mortality, severe skin hyperpigmentation, defective hair follicle morphogenesis and widespread epithelial dysplasia (34). Furthermore, conditional TPP1 inhibition may accelerate telomere shortening in the skin, which indicates that TPP1 is involved in telomere maintenance. The epithelial pathologies that can be found in TPP1-deficient mice are comparable with epithelial pathologies in human diseases associated with mutations in telomerase-related genes and the presence of dysfunctional telomeres (34). In conclusion, the results of previous investigations into TPP1 deficiency in mouse models indicate that TPP1 performs a dual role in telomere protection and elongation, thus preserving telomere function and preventing the early onset of degenerative pathologies in mice $(9,34)$.

In a human study, Augereau et al (38) compared 23 patients with newly diagnosed early-stage B-cell chronic lymphocytic leukemia (CLL) with 12 healthy donors. A significant increase in the recruitment of DNA-damage factors to the telomeres was detected, suggesting telomere dysfunction at the early stage of the disease. Notably, the presence of dysfunctional telomeres did not correlate with telomere shortening or chromatin mark deregulation, but did correlate with the downregulation of the shelterin genes $A C D$ and TINF2. Augereau et al proposed that telomeric deprotection in the early stages of CLL is a consequence of telomere alteration, in addition to telomere shortening. The authors observed the damage that occurred at an early stage of the disease and suggested that it contributed to the early step of malignant transformation (38).

In the present study, the gene dysregulation observed was similar to that detected by Augereau et al (38). According to our hypothesis, the downregulation of $A C D / \mathrm{TPP} 1$ occurs prior to the first stage of malignant transformation, i.e. during the risk factor-enhancing immunosuppressive therapy. Immunosuppressive therapy is considered to be a major cause of increased cancer incidence following organ transplantation. A number of aspects of immunosuppressive therapy are considered risk factors, including the type of immunosuppression, the number of immunosuppressive drugs and the dose administered (4). The use of lymphocyte-depleting antibodies has been demonstrated to increase the risk of virally-induced malignancies, while lower-dose CsA regimens were associated with a reduced incidence of tumors compared with standard therapy (1). The patients enrolled in the present study received a triple-immunosuppression scheme consisting of CsA, MMF or mycophenolate sodium and prednisone; the drugs were administered at a similar dose on the basis of the patient weight and drug serum level. Thus the study group was homogenous in case of type and dosage of taken immunosupresive therapy. The molecular mechanism of carcinogenesis in immunosuppressive therapy remains unclear. Notably, certain drugs appear to promote oncogenesis by mechanisms independent of their immunosuppressive effects (39) and are considered to be major additional risk factors for cancer development (1). Among these drugs, calcineurin inhibitors have been associated with the development of post-transplant malignancies, such as lymphoma and solid organ tumors. PBMCs from renal transplant recipients undergoing CsA therapy have been observed to exhibit a reduced ability to repair radiation-induced DNA damage (40), which may contribute to carcinogenesis. The CsA-induced production of cytokines, such as transforming 
growth factor- $\beta$ and/or vascular endothelial growth factor, may additionally promote carcinogenesis $(39,41,42)$.

Despite the risks associated with certain immunosuppressive drugs, others have exhibited antiproliferative activity. Mammalian target of rapamycin (mTOR) inhibitors, e.g. rapamycin, play a protective role in cancer development (43); however, since mTOR inhibitors were not used in the present study, their mechanism will not be expanded upon. MMF has also been demonstrated to exert a protective effect in immunosuppression regimens in population analyses performed in transplant recipients $(39,44)$. Administration of MMF has been shown to result in a clear antitumor effect against leukemia and lymphoma $(45,46)$ and against colon and prostate carcinoma cells (47). Additionally, MMF suppresses glycosylation and the expression of a number of adhesion molecules in solid tumor dissemination (47), while inhibiting the adhesion of colon adenocarcinoma cells to endothelial cells $(39,48)$.

In the present study the transplant patients were administered a typical but complex immunosuppression regimen comprising CsA and antiproliferative MMF with prednisone in low doses that were oncogenically neutral. The results of the present study suggest that a cumulative pro-oncogenic effect is associated with immunosuppressive therapy, in addition to protection by MMF. Furthermore, the molecular alterations may affect telomere maintenance at an early stage when, despite the stability of the telomere length and telomerase remaining at a low level, the assembly and stabilization of the telosome/shelterin protein complex is disturbed, mediating the access of telomerase to the telomere.

To the best of our knowledge, the present study provides the first evidence of the dysregulation of telomere homeostasis, in association with alterations of the telosome/shelterin complex, in patients receiving immunosuppressive therapy. The results of this study demonstrate that the downregulation of the $A C D$ gene (TPP1 protein) may increase the capacity for cell immortalization, despite normal levels of other key telomere maintenance factors, in patients undergoing immunosuppressive therapy following renal transplantation. Future investigation into $A C D /$ TPP1 may aid the search for early clinical markers and/or therapeutic targets for cancer in patients following organ transplantation.

\section{Acknowledgements}

This study was supported by grants from the Medical University of Silesia (nos. KNW-1-043/09 and KNW-1-047/D/1/0; Silesia, Poland). The authors express their gratitude to $\mathrm{Dr}$ Joanna Zywiec, Dr Bozena Kaminska-Trus and Dr Sylwia Kosiorz (Department of Internal Medicine, Diabetology and Nephrology, Medical University of Silesia) for their assistance in patient enrollment.

\section{References}

1. Alberú J: Clinical insights for cancer outcomes in renal transplant patients. Transplant Proc 42 (9 Suppl): S36-S40, 2010.

2. Briggs JD: Causes of death after renal transplantation. Nephrol Dial Transplant 16: 1545-1549, 2001.

3. Kasiske BL, Snyder JJ, Gilbertson DT and Wang C: Cancer after kidney transplantation in the United States. Am J Transplant 4: 905-913, 2004
4. First MR and Peddi VR: Malignancies complicating organ transplantation. Transplant Proc 30: 2768-2770, 1998.

5. Maser RS and DePinho RA: Connecting chromosomes, crisis and cancer. Science 297: 565-569, 2002.

6. Cheung AL and Deng W: Telomere dysfunction, genome instability and cancer. Front Biosci 13: 2075-2090, 2008.

7. Grady WM: Genomic instability and colon cancer. Cancer Metastasis Rev 23: 11-27, 2004.

8. de Lange T: Shelterin: The protein complex that shapes and safeguards human telomeres. Genes Dev 19: 2100-2110, 2005.

9. Martínez P and Blasco MA: Role of shelterin in cancer and aging. Aging Cell 9: 653-666, 2010.

10. Lu W, Zhang Y, Liu D, Songyang Z and Wan M: Telomeres-structure, function and regulation. Exp Cell Res 319: 133-141, 2013.

11. Artandi SE and DePinho RA: Telomeres and telomerase in cancer. Carcinogenesis 31: 9-18, 2010.

12. Kong CM, Lee XW and Wang X: Telomere shortening in human diseases. Febs J 280: 3180-3193, 2013.

13. DePinho RA and Polyak K: Cancer chromosomes in crisis. Nat Genet 36: 932-934, 2004.

14. Hanahan D and Weinberg RA: The hallmarks of cancer. Cell 100: 57-70, 2000.

15. Harley CB, Futcher AB and Greider CW: Telomeres shorten during ageing of human fibroblasts. Nature 345: 458-460, 1990.

16. Wenz C, Enenkel B, Amacker M, Kelleher C, Damm K and Lingner J: Human telomerase contains two cooperating telomerase RNA molecules. Embo J 20: 3526-3534, 2001.

17. Mergny JL, Riou JF, Mailliet P, Teulade-Fichou MP and Gilson E: Natural and pharmacological regulation of telomerase. Nucleic Acids Res 30: 839-865, 2002.

18. Kim NW, Piatyszek MA, Prowse KR, et al: Specific association of human telomerase activity with immortal cells and cancer. Science 266: 2011-2015, 1994.

19. Hiyama E and Hiyama K: Telomerase as tumor marker. Cancer Lett 194: 221-233, 2003.

20. Calado R and Young N: Telomeres in disease. F1000 Med Rep 4: 8, 2012.

21. Witkowska A, Gumprecht J, Glogowska-Ligus J, et al: Expression profile of significant immortalization genes in colon cancer. Int J Mol Med 25: 321-329, 2010.

22. Rostkowska-Nadolska B, Kapral M, Fraczek M Kowalczyk M, Gawron W and Mazurek U: Transcriptional activity of genes-encoding kinin $\mathrm{B} 1$ and $\mathrm{B} 2$ receptors and kinin-dependent genes in nasal polyps. Adv Med Sci 54: 211-220, 2009

23. Strzalka-Mrozik B, Stanik-Walentek A, Kapral M, et al: Differential expression of transforming growth factor-beta isoforms in bullous keratopathy corneas. Mol Vis 16: 161-166, 2010.

24. Berezowski P, Strzalka-Mrozik B, Forminska-Kapuscik M, et al: Posttraumatic temporal TGF-beta mRNA expression in lens epithelial cells of paediatric patients. Folia Biol (Praha) 58: 24-29, 2012.

25. Kapral M, Wawszczyk J, Jurzak M, Hollek A and Weglarz L: The effect of inositol hexaphosphate on the expression of selected metalloproteinases and their tissue inhibitors in IL-1 $\beta$-stimulated colon cancer cells. Int J Colorectal Dis 27: 1419-1428, 2012 .

26. Pfaffl MW, Horgan GW and Dempfle L: Relative expression software tool (REST) for group-wise comparison and statistical analysis of relative expression results in real-time PCR. Nucleic Acids Res 30: e36, 2002.

27. Chen LY, Liu D and Songyang Z: Telomere maintenance through spatial control of telomeric proteins. Mol Cell Biol 27: 5898-5909, 2007.

28. Cong YS, Wright WE and Shay JW: Human telomerase and its regulation. Microbiol Mol Biol Rev 66: 407-425, 2002.

29. Ducrest AL, Szutorisz H, Lingner J and Nabholz M: Regulation of the human telomerase reverse transcriptase gene. Oncogene 21: 541-552, 2002.

30. Xu L, Li S and Stohr BA: The role of telomere biology in cancer. Annu Rev Pathol 8: 49-78, 2013.

31. Kibe T, Osawa GA, Keegan CE and de Lange T: Telomere protection by TPP1 is mediated by POT1a and POT1b. Mol Cell Biol 30: 1059-1066, 2010.

32. Xin H, Liu D, Wan M, et al: TPP1 is a homologue of ciliate TEBP-beta and interacts with POT1 to recruit telomerase. Nature 445: 559-562, 2007. 
33. Zhong FL, Batista LF, Freund A, Pech MF, Venteicher AS and Artandi SE: TPP1 OB-fold domain controls telomere maintenance by recruiting telomerase to chromosome ends. Cell 150 : 481-494, 2012.

34. Tejera AM, Stagno d'Alcontres M, Thanasoula M, et al: TPP1 is required for TERT recruitment, telomere elongation during nuclear reprogramming and normal skin development in mice. Dev Cell 18: 775-789, 2010.

35. Chen LY, Redon S and Lingner J: The human CST complex is a terminator of telomerase activity. Nature 488: 540-544, 2012.

36. Nakashima M, Nandakumar J, Sullivan KD, Espinosa JM and Cech TR: Inhibition of telomerase recruitment and cancer cell death. J Biol Chem 288: 33171-33180, 2013.

37. Yang L, Wang W, Hu L, et al: Telomere-binding protein TPP1 modulates telomere homeostasis and confers radioresistance to human colorectal cancer cells. PLoS One 8: e81034, 2013.

38. Augereau A, T'Kint de Roodenbeke C, Simonet T, et al: Telomeric damage in early stage of chronic lymphocytic leukemia correlates with shelterin dysregulation. Blood 118: 1316-1322, 2011.

39. Buell JF, Gross TG and Woodle ES: Malignancy after transplantation. Transplantation 80 (2 Suppl): S254-S264, 2005.

40. Herman M, Weinstein T, Korzets A, et al: Effect of cyclosporin A on DNA repair and cancer incidence in kidney transplant recipients. J Lab Clin Med 137: 14-20, 2001.

41. Hojo M, Morimoto T, Maluccio M, et al: Cyclosporine induces cancer progression by a cell-autonomous mechanism. Nature 397: $530-534,1999$.
42. Shihab FS, Bennett WM, Isaac J, Yi H and Andoh TF: Nitric oxide modulates vascular endothelial growth factor and receptors in chronic cyclosporine nephrotoxicity. Kidney Int 63: 522-533, 2003.

43. Koehl GE, Andrassy J, Guba M, et al: Rapamycin protects allografts from rejection while simultaneously attacking tumors in immunosuppressed mice. Transplantation 77: 1319-1326, 2004.

44. Dharnidharka VR, Ho PL, Stablein DM, Harmon WE and Tejani AH: Mycophenolate, tacrolimus and post-transplant lymphoproliferative disorder: A report of the North American Pediatric Renal Transplant Cooperative Study. Pediatr Transplant 6: 396-399, 2002.

45. Ohsugi Y, Suzuki S and Takagaki Y: Antitumor and immunosuppressive effects of mycophenolic acid derivatives. Cancer Res 36: 2923-2927, 1976

46. Carter SB, Franklin TJ, Jones DF, et al: Mycophenolic acid: an anti-cancer compound with unusual properties. Nature 223: 848-850, 1969.

47. Engl T, Makarević J, Relja B, et al: Mycophenolate mofetil modulates adhesion receptors of the betal integrin family on tumor cells: Impact on tumor recurrence and malignancy. BMC Cancer 5: 4, 2005.

48. Leckel K, Beecken WD, Jonas D, et al: The immunosuppressive drug mycophenolate mofetil impairs the adhesion capacity of gastrointestinal tumour cells. Clin Exp Immunol 134: 238-245, 2003. 